\title{
Network Connectivity based Topology Control for Mobile Ad Hoc Networks
}

\author{
T.S.Asha \\ Associate Professor \\ Department of ECE \\ NSS College of Engineering, Palakkad, Kerala
}

\author{
N.J.Muniraj, PhD. \\ Principal \\ Tejaa Shakthi Institute of Technology, \\ Coimbatore
}

\begin{abstract}
Energy consumption and network connectivity are two of the important research issues that are yet to be resolved in mobile ad hoc networks (MANETs). However, only a few topology control methods (e.g. [1]) take into account the low interference as a goal of the methods. Some researchers tried to indirectly reduce the interference by reducing the transmission power or by devising low degree topologies, but none of those protocols can guarantee low interference. In this research work, we propose Network Connectivity based Topology Control (NCTC) to make the correct the balance between interference and energy in order to improve the network lifetime of networks. It consists of two phases. In first phase, the reduction of interference is achieved. In second phase, efficient topology control based on energy constraint is proposed to extend the network lifetime of networks. By using the extensive simulation results using Network Simulator (NS2), the proposed scheme NCTC achieves better network lifetime, packet delivery ratio, less overhead and end to end delay than the existing schemes.
\end{abstract}

\section{General Terms}

Network Connectivity, Topology control.

\section{Keywords}

MANET, Interference, network connectivity, network lifetime, packet delivery ratio, end to end delay, overhead.

\section{INTRODUCTION}

\subsection{Mobile Ad Hoc Networks (MANETs)}

Mobile ad-hoc network is an independent system of mobile nodes connected by wireless links forming a short, live, onthe-fly network even when access to the Internet is unavailable. Nodes in MANETs generally operate on low power battery devices. These nodes can function both as hosts and as routers. As a host, nodes function as a source and destination in the network and as a router, nodes act as intermediate bridges between the source and the destination giving store-and-forward services to all the neighbouring nodes in the network. Easy deployment, speed of development, and decreased dependency on the infrastructure are the main reasons to use ad-hoc network.

\subsection{The topology control problem in MANET}

In mobile ad hoc wireless communication, each node of the network has a potential of varying the topology through the adjustment of its power transmission in relation to other nodes in the neighborhood. In contrast, wired networks have fixed established pre-configured infrastructure with centralized network management system structure in place. Therefore, the fundamental reason for the topology control scheme in MANET is to provide a control mechanism that maintains the network connectivity and performance optimization by prolonging network lifetime and maximizing network throughput. A MANET topology can depend on uncontrollable factors such as node mobility, weather, interference, noise as well as controllable factors such as transmission power, directional antennas and multi-channel communications.

A bad topology can impact negatively on the network capacity by limiting spatial reuse capability of the communication channel and also can greatly undermine the robustness of the network. Where network capacity means the bandwidth and ability for it to be used for communication. A network partitioning can occur in a situation where the network topology becomes too sparse. Similarly, a network which is too dense is prone to interference at the medium access (MAC) layer, the physical layer of the network. So the network should neither be too dense nor too sparse for efficient communication amongst nodes to take place.

\subsection{Problem Definition}

The problem identified in contemporary research literature pertaining to topology control in MANET is that most of the topology control algorithms do not achieve reliable and guaranteed network connectivity.

\section{RELATED WORK}

Dalu et.al [1] proposed pursue mobility model which does not maintain the topology without any control message. There is no need to change routing table as the connectivity of the network is maintained all through. No node ever diverges out of the communication range. Even if any pursuer node goes out of the maximum allowable safe distance, communication with the target node would not be hampered, since the communication range is higher than the maximum allowable safe distance. Here the system never becomes static as a whole and hence greater efficiency is achieved in terms of time.

Jie $\mathrm{Wu}$ and Fei Dai [2] proposed mobility-sensitive topology control method that extends many existing mobilityinsensitive protocols. Two mechanisms are introduced: consistent local views that avoid inconsistent information, and delay and mobility management that tolerate outdated information. This method is based on two mechanisms: local view synchronization based on synchronous or asynchronous "Hello" messages, and buffer zone created by slightly increasing the actual transmission range. These two mechanisms ensure the connectivity of both logical topology and effective topology, two notions proposed for topology control in dynamic networks. 
Venkatesan [3] presented a distributed algorithm for finding a minimal connected dominating set. Our algorithm has been implemented and numerous simulation experiments have been conducted. In the simulated topologies, the algorithm converges very quickly. The worst case for our algorithm consists of all nodes being arranged symmetrically and the degree of shortest paths connectivity provided by each node is the same.

Quansheng Guan [5] proposed a distributed prediction-based cognitive topology control (PCTC) scheme to provision cognition capability to routing in CR-MANETs. PCTC is a middleware-like cross-layer module residing between CRmodule and routing. It uses cognitive link availability prediction, which is aware of the interference to primary users and user mobility, to predict the available duration of links. Based on the link prediction, PCTC captures the dynamic changes of the topology and constructs an efficient and reliable topology, which is aimed at mitigating rerouting frequency and improving end-to-end network performance such as throughput and delay.

Suchismita routa [6] proposed a two-phase DBSS protocol which deals with topology control and provides the mechanism to reduce overall energy consumption in networks. They discussed on how topology of deployment of nodes plays an important role regarding energy conservation. In DBSS protocol it takes farthest node in its transmission range for routing. That node is geographically closer to the destination. The number of nodes in packet transferring is less. They have addressed how the topology of the network can be adjusted by controlling the transmission power. In this work the node in the farthest transmission range will take part in routing and the node that was geographically closer to the destination node is the candidate. Energy conservation is based on sleep based approaches. The energy was conserved by controlling a set of neighbor to which the node communicates.

Abhishek majumder [7] presented an energy and mobility aware clustering approach. The clustering approach is incorporated in a DSR like protocol for routing in MANET to evaluate the performance improvement gained due to clustering using proposed approach. Rate of cluster head changes, throughput of the network, delay and routing overhead is evaluated using NS2

Quansheng Guan [8] explored Capacity-Optimized Cooperative (COCO) topology control scheme to improve the network capacity in MANETs by jointly considering both upper layer network capacity and physical layer cooperative communications. They have introduced physical layer cooperative communications, topology control, and network capacity in MANETs. To improve the network capacity of MANETs with cooperative communications.

Atsushi yoshinari et.al [9] proposed mechanism with an adopted topology control technique, based on a localized algorithm, can maintain local connectivity which results in keeping global network connectivity although the network is dynamic. In the proposed topology update mechanism, the update interval in each node is determined based on the transmission range and mobility information of its adjacent nodes so that the network connectivity is guaranteed.

Gaurav Srivastava [10] compared several topology algorithms like centralised and distributed topology control algorithms. They also provided a comparison of these algorithms and suggest which algorithms may perform best. They also gave comment on the partitioning, routing, scheduling and latency issues that may arise due to topology adaptations in a mobile ad-hoc network.

Srinivas Rao et.al [11] proposed power management schemes looks into two directions. First is to balance power consumption during data transfer and secondly to reduce the power consumed in case of a route failure. By balancing power consumption we can avoid the death of some critical nodes caused by excessive power consumption. Reducing power consumption intends to prolong the lifetime of each node which in turn extends the lifetime of the entire network. Each approach proposed in the following sections improves the network's performance either by balancing the power across the network or by reducing the power consumed by the nodes across the network.

Hiroki Nishiyama et.al [12] proposed a dynamic method is proposed to effectively employ k-edge connected topology control algorithms in MANETs. The proposed method automatically determines the appropriate value of $\mathrm{k}$ for each local graph based on local information while ensuring the required connectivity ratio of the whole network. The results show that the dynamic method can enhance the practicality and scalability of existing k-edge connected topology control algorithms while guaranteeing the network connectivity.

The paper is organized as follows. The Section 1 describes introduction about MANET, topology control problem in MANET. Section 2 deals with the previous work which is related to the topology control. Section 3 is devoted for the implementation of Network Connectivity based Topology Control. Section 4 describes the performance analysis and the last section concludes the work.

\section{IMPLEMENTATION OF NETWORK CONNECTIVITY BASED TOPOLOGY CONTROL SCHEME}

In the proposed network connectivity topology control, there are two phases involved. In first phase, we aim to minimize the maximum interference. In second phase, we propose the efficient topology control approach which consists of minimal weight estimation and exchanging information, topology estimation.

\subsection{Interference Reduction}

Please use a 9-point Times Roman font, or other Roman font with serifs, as close as possible in appearance to Times Roman in which these guidelines have been set. The goal is to have a 9-point text, as you see here. Please use sans-serif or non-proportional fonts only for special purposes, such as distinguishing source code text. If Times Roman is not available, try the font named Computer Modern Roman. On a Macintosh, use the font named Times. Right margins should be justified, not ragged.

\subsection{Efficient Topology Control Approach}

In this section, we propose the NCTC algorithm to build energy-efficient topology for wireless multihop networks. We model the topology of a wireless network with each node using its maximal transmission power as an undirected graph $\mathrm{G}=(\mathrm{L}, \mathrm{R})$ in the two dimensional plane, where $\mathrm{L}=\left\{1_{1}, \mathrm{l}_{2}, \ldots\right.$, $1_{n}$ \} is the set of nodes in the network and $E$ is the set of bidirectional links. The network may be heterogeneous, and hence each node $\mathrm{L}_{\mathrm{i}}$ may have its own transmission power $\mathrm{p}_{\mathrm{t}}$ which can be adjusted by itself. Here only bidirectional links 
are concerned. Therefore the bidirectional link $\left(l_{i}, l_{j}\right), \quad R$ implies that both $\mathrm{L}_{\mathrm{i}}$ and $\mathrm{L}_{\mathrm{j}}$ are covered by each other. We define the physical neighbor set of each node $\mathrm{L}_{\mathrm{i}}$ as

$$
N T_{s}^{t}=\left\{l_{k} \mid\left(l_{i}, l_{k}\right) \in R(G)\right\}
$$

In NCTC, each bidirectional link is assigned a weight which can be derived from the weight function w. Thus the weight of a link $\left(1_{i}, 1_{j}\right)$ can be expressed by $w(i, j)$.

We use link weight to represent the energy consumption required in the transmission along a link and use path weight to represent the sum of all link weights of a path. Therefore, we define the minimal energy path as the path with the minimal path weight

The computation of $w(i, j)$ usually relates only to $l_{i}$ and $l_{j}$, at most to their neighbors. This makes it possible that each node runs NCTC only according to the locally collected information. However, the algorithm will not miss any logical neighbor which is placed on some minimal energy path in the original network. Therefore, NCTC preserves minimal energy paths in the main topology

\subsubsection{Minimal weight estimation}

Each node in this step locally collects the information needed in the weight calculations for all links associated with it, and calculates link weights. Note that different energy model may require different information in the calculations. For the design of the weight function $\mathrm{w}$, we suggest that it should have the same value for both directions of a link. This makes sense since communication always consists of transmissions (including data transmissions and control message transmissions) in both directions, and furthermore this ensures that for any two nodes, they have the same mini mal energy path connecting them. However, in some energy model the two endpoints of a link $\left(l_{i}, l_{j}\right)$ may derive different weights (called unidirectional link weight, and designed as

$$
w(i, j)=(\vec{w}(i, j)+\vec{w}(j, i) / 2
$$

Here each node derives the unidirectional link weights.

\subsubsection{Link Weight Information Exchange}

In this step, each node $l_{i}$ exchanges the unidirectional link weight information derived in the first step with its 1-hop neighbors by broadcasting using its maximal transmission power pi max. After that, $1_{\mathrm{i}}$ knows all the weights calculated at the nodes in the set of $\left\{1_{i}\right\} \quad U$ $N T_{s}^{t}=\left\{l_{k} \mid\left(l_{i}, l_{k}\right) \in R(G)\right\}$.

If there is a bidirectional link $\left(1_{x}, 1_{y}\right), l_{i}$ can derive $w(x, y)$. Two points should be mentioned. First, node $l_{i}$ may receive $w(j, i)$ from node $l_{j}$ while $l_{j}$ is out of its range. In this case, link $\left(l_{j}, l_{i}\right)$ is not considered by $l_{i}$. The information exchange in this step is avoidable if each node vi can obtain the information required to calculate the unidirectional link weights for all the nodes in $N T_{s}^{t}$. In this case, each node does all the weight calculations for all links. Although this approach needs more computation time and stricter preconditions, it reduces the number of information exchanges to one.

\subsubsection{Local Topology Construction}

In this step, each node $l_{i}$ computes the local shortest path connecting it to every node $\mathrm{l}_{\mathrm{j}} \in N T_{s}^{t}$. according to the derived link weights. The Dijkstra's algorithm can be employed if there is no negative link weight, and the time complexity varies from $\mathrm{O}\left(\left|N T_{s}^{t} \cdot\right|\right)$ to $\mathrm{O}\left(\left|E N T_{s}^{t}\right| \log \mid\right.$ $N T_{s}^{t} \mid$ ) depending on the implementation of the algorithm where $E N T_{s}^{t}$. is the set of all bidirectional links whose endpoints are in $N T_{s}^{t}$. If negative link weights exist, then the Bellman-Ford algorithm can be used. .Note that in some networks, transmissions along a link may reduce the power consumptions of the nearby nodes by using some strategy such as turning their radios off. In this case, the link may have a negative weight. Denote the local shortest path connecting $1_{x}$ to $1_{\mathrm{y}} \in N T_{s}^{t}$ as

$$
P^{x, y}=\left(l_{p 0} x, y=l_{i}, l_{p 1} x, y \ldots \ldots l_{p_{n-1}} x, y, l_{p_{n}} x, y=l_{y}\right)
$$

logical neighbor set $N S_{s}^{q}$ can be represented by

$$
N S_{s}^{q}=\left\{l_{p 1} i, x \mid l_{x} \in \cdot \mathrm{NT}_{\mathrm{s}}^{\mathrm{t}}\right\}
$$

That is, all the second nodes on the paths compose the logical neighbor set. Note that the path is bidirectional since every link on the path is bidirectional. However, the path pathj,I is not the reverse of path $i, j$ Another point we should mention is that not every neighbor close to $l_{i}$ is its logical neighbor and meanwhile not every logical neighbor of $l_{i}$ is close to it since the short link is not always energy efficient according to some realistic energy model. Through the construction of the local shortest paths, each node can derive a local route table which is described as

\begin{tabular}{|l|l|l|l|l|l|}
\hline Destination & $\begin{array}{l}\text { Next } \\
\text { hop }\end{array}$ & $\begin{array}{l}\text { Link } \\
\text { weight }\end{array}$ & $\begin{array}{l}\text { Hop } \\
\text { count }\end{array}$ & IR & CRC \\
\hline
\end{tabular}

\section{Fig.1.Proposed Packet format}

In fig1, IR describes Interference ratio of each link. Cyclic Redundancy Check (CRC) for error correction and detection. Each physical neighbor(the destination) has an entry in the table. The link weight represents the weight of the link connecting the current node and the next hop. It can be used by upper level routing algorithm to find a least weighted path. The network topology under NCTC is all the nodes in L and their individually perceived logical neighbor relations.

\section{PERFORMANCE ANALYSIS}

Network Simulator (NS) is an event driven network simulator developed at UC Berkeley that simulates variety of IP networks. It implements network protocols such as TCP and UPD, traffic source behavior such as FTP, Telnet, Web, CBR and VBR, router queue management mechanism such as Drop Tail, RED and CBQ, routing algorithms such as Dijkstra, and more. NS also implements multicasting and some of the MAC layer protocols for LAN simulations.Currently, NS (version 2) 
written in $\mathrm{C}++$ and $\underline{\mathrm{OTcl}}$ (Tcl script language with Objectoriented extensions developed at MIT) is available.

We use NS3 to simulate our proposed algorithm. In our simulation, 200 mobile nodes move in a 1000 meter $\mathrm{x}$ 1000 meter square region for 80 seconds simulation time. All nodes have the same transmission range of 300 meters. The simulated traffic is Constant Bit Rate (CBR). Our simulation settings and parameters are summarized in table 1 .

\begin{tabular}{|l|l|}
\hline No. of Nodes & 200 \\
\hline Area Size & $1000 \times 1000$ \\
\hline Mac & 802.11 \\
\hline Radio Range & $300 \mathrm{~m}$ \\
\hline Simulation Time & $50 \mathrm{sec}$ \\
\hline Traffic Source & CBR \\
\hline Packet Size & \multicolumn{1}{|c|}{512 bytes } \\
\hline Mobility Model & Random Way Point \\
\hline Protocol & AODV \\
\hline Packet rate & $6 p k t s / s e c$ \\
\hline
\end{tabular}

\section{Performance Metrics}

We evaluate mainly the performance according to the following metrics.

Control overhead: The control overhead is defined as the total number of routing control packets normalized by the total number of received data packets.

Packet Delivery Ratio: The packet delivery ratio (PDR) of a network is defined as the ratio of total number of data packets actually received and total number of data packets transmitted by senders.

Node degree: It is the important metric to evaluate the performance of topology control algorithms. If the node degree is higher, it indicates that higher collision will be. So value of node degree should be kept small.

Network connectivity ratio: It determines the nodes are connected in the intermediate region. It should be kept small while varying the average speed.

End-to-End Delay: The End-to-End delay is defined as the difference between two time instances: one when packet is generated at the sender and the other, when packet is received by the receiving application.

The simulation results are presented in the next part. We compare our proposed algorithm NCTC with DM [12] in presence of topology control environment.

Figure 2 shows the results of connectivity ratio for varying the mobility from 5 to 25 . From the results, we can see that NCTC scheme has slightly lower connectivity ratio than the DM method because of light weight calculations.

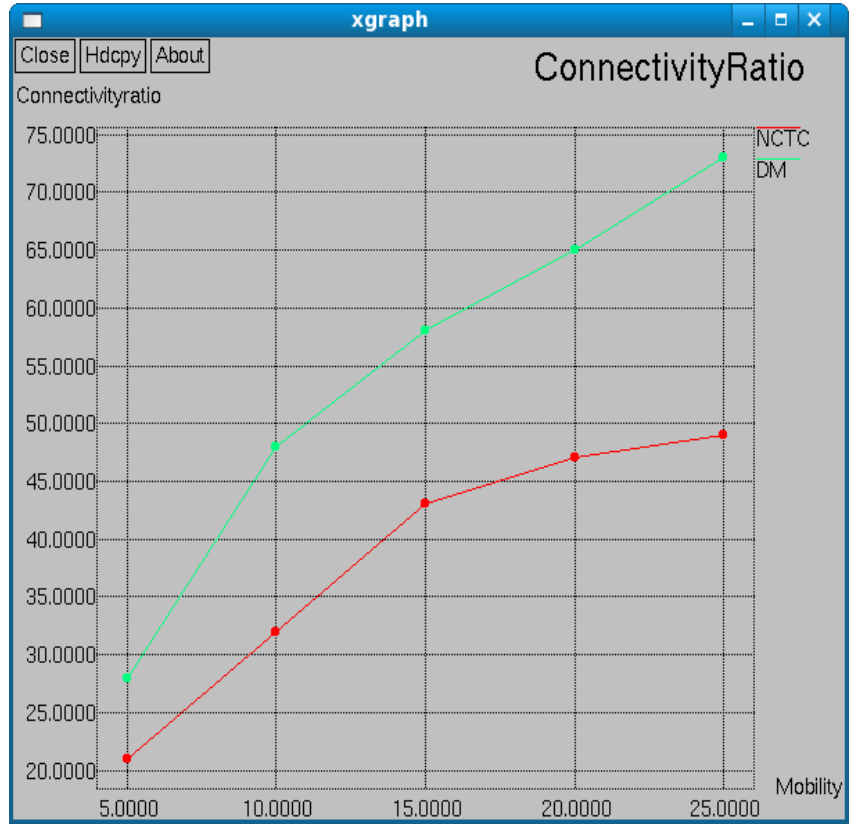

Fig.2. Mobility Vs Connectivity Ratio

Fig. 3, presents the comparison of node degree It is clearly shown that the node degree of NCTC has low overhead than the DM

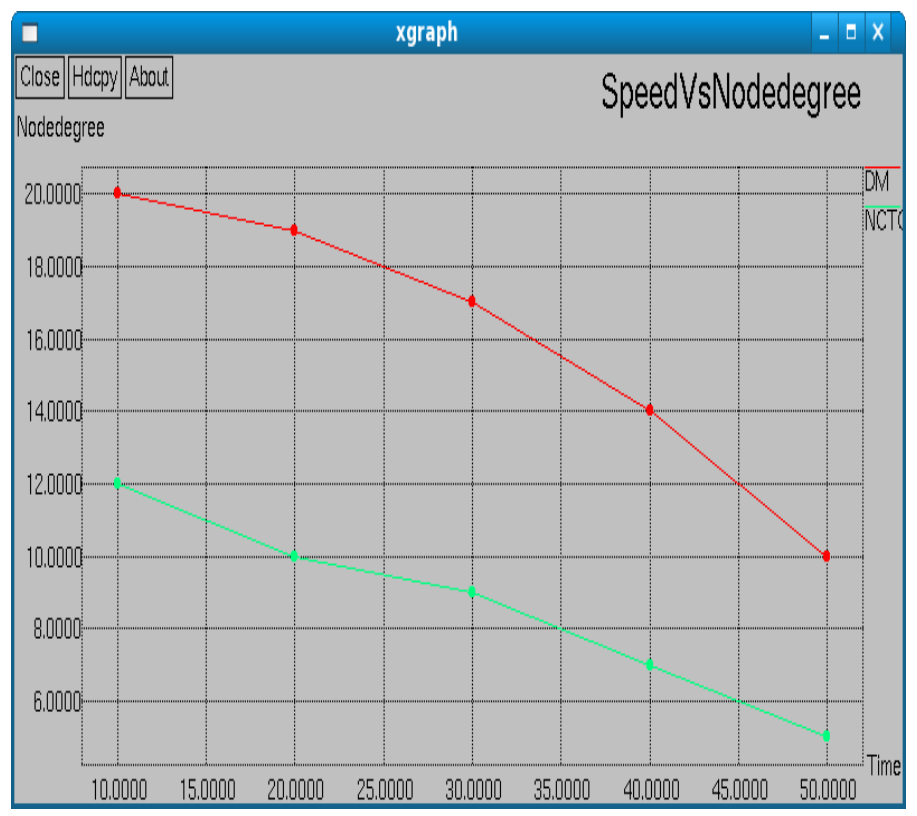

Fig. 3. Speed Vs Node degree

Figure 4 shows the results of Time Vs End to end delay. From the results, we can see that NCTC scheme has slightly lower delay than the DM scheme because of stable routines. 


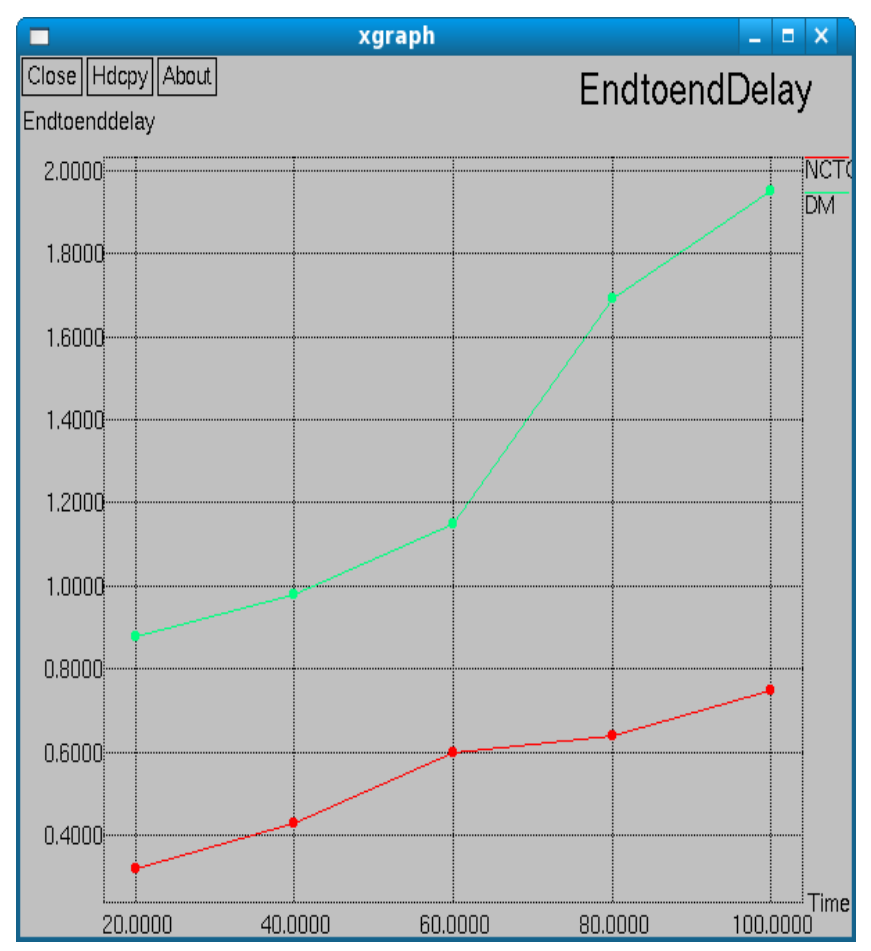

Fig. 4. Time Vs End to end delay

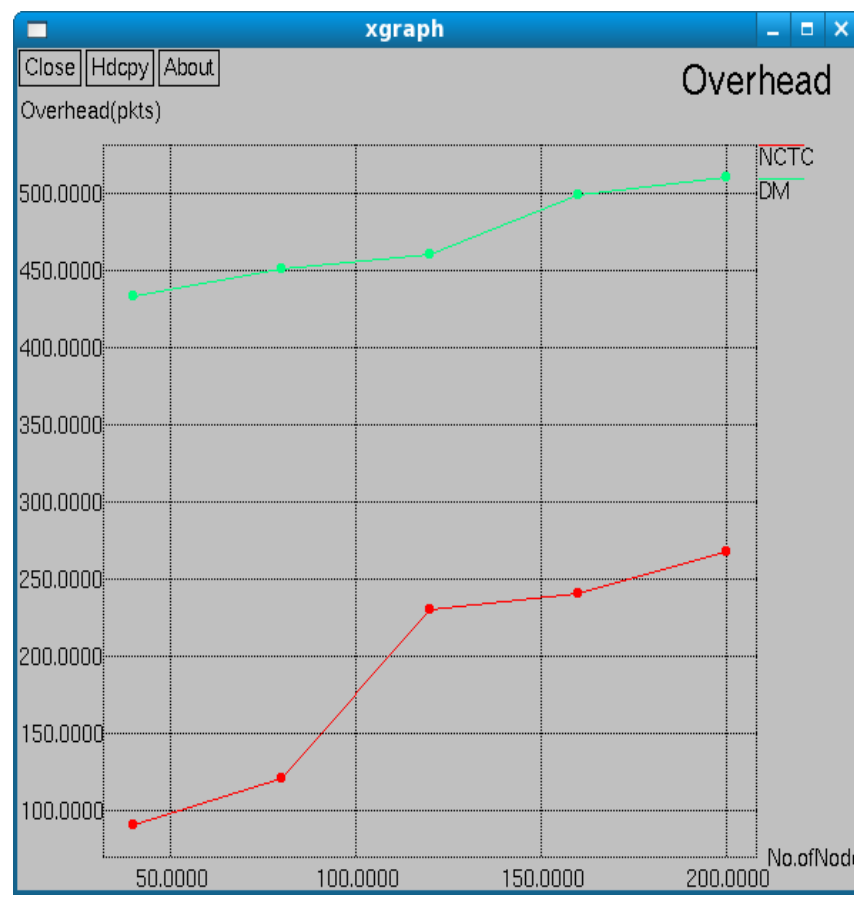

Fig.5.No. of Nodes Vs Overhead

Fig. 5, presents the comparison of overhead while varying the nodes from 0 to 200 . It is clearly shown that the of NCTC has low overhead than the DM method.

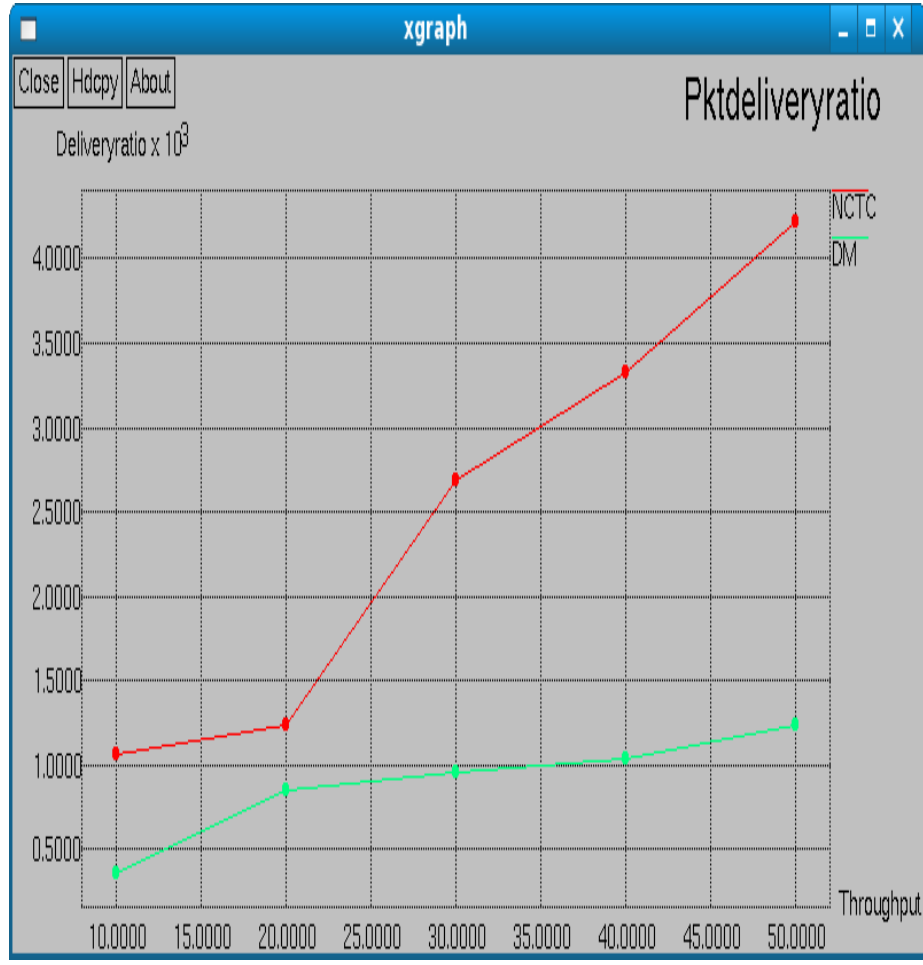

Fig.6. Throughput Vs Packet Delivery Ratio

Figure 8 show the results of average packet delivery ratio for the throughput $10,20 \ldots 50$ for the 200 nodes scenario. Clearly our NCTC scheme achieves more delivery ratio than the DM scheme since it has both topology control features.

\section{CONCLUSION}

Mobile nodes are communicating without any access point in MANETs. Due to the uncontrolled topologies, the more interference and more energy consumption is introduced in the networks which leads to less performance of network connectivity. In this paper, we have introduced the network connectivity based topology control to make the correct balance between the energy efficiency and interference to improve the network connectivity. In first phase, we have achieved low interference using based on the recommendation of neighbor nodes. In second phase, the efficient topology control scheme is introduced to extend the network lifetime of MANET. By simulation results we have shown that the NCTC achieves good packet delivery ratio, better network lifetime while attaining low delay, overhead, while varying the number of nodes, node velocity and mobility.

\section{REFERENCES}

[1] S. S. Dalu, , M. K. Naskar and C. K. Sarkar," Implementation of a Topology Control Algorithm for Mobile Ad hoc Networks Using Pursue Mobility Model", Journal of Applied Computer Science, no.2 (2) /2008, Suceava, pp.14-20.

[2] Jie $\mathrm{Wu}$ and Fei Dai, "Mobility-Sensitive Topology Control in Mobile Ad Hoc Networks", pp.1-10.

[3] S. Venkatesan and C. David Young , "A Distributed Topology Control Algorithm for MANETS”, pp.1-7.

[4] S. Karunakaran and P. Thangaraj, "Topology Control Using Efficient Power Management", Journal of Computer Science 7 (4): 561-567, 2011, ISSN 15493636, pp.561- 567. 
[5] Quansheng Guan, F.RichardYu, Shengming Jiang and Gang Wei, "Prediction-Based Topology Control and Routing in Cognitiv e Radio Mobile Ad Hoc Networks", IEEE Transactions On Vehicular Technology, Vol. 59, No. 9, November 2010, pp.4443-4452.

[6] Suchismita Rout, Ashok Kumar Turuk and Bibhudatta Sahoo, "Energy Aware Routing Protocol in MANET using Power Efficient Topology Control Method", International Journal of Computer Applications (0975 8887), Volume 43- No.5, April 2012, pp.33-42.

[7] Abhishek Majumder and Nityananda Sarma, "DEMAC: A Cluster-Based Topology Control for Ad Hoc Networks", International Journal of Computer Science Issues, Vol. 7, Issue 5, September 2010, pp.8288.

[8] Quansheng Guan, F.RichardYu, Shengming Jiang and Victor C.M.Lung, "Topology Control in Mob,ile Ad hoc Networks With Cooperative Communications", IEEE Wireless communications, 2012, pp.2-7.

[9] Atsushi Yoshinari, Hiroki Nishiyama, Nei Kato, and Dan Keun Sung, "Dynamic Topology Update Mechanism in Local Tree-based Relialble Topology (LTRT) based MANETs," IEEE International Conference on Communications (ICC 2012), Ottawa, Canada, Jun. 2012, pp.1-6.

[10] Gaurav Srivastava, Paul Boustead and Joe F.Chicharo, "A Comparison of Topology Control Algorithms for Adhoc Networks",

[11] Dr D Srinivas Rao, Jooby Ann Thomas and Sake Pothalaiah, "QoS through Power Control in MANETs using AODV Protocol", International Journal of Computer Applications (0975 - 8887) , Volume 31No.9, October 2011, pp.23-29.

[12] Hiroki Nishiyama, Thuan Ngo, Nirwan Ansari, and Nei Kato, "On Minimizing the Impact of Mobility on Topology Control in Mobile Ad Hoc Networks," IEEE
Transactions on Wireless Communications, vol.11, no.3, pp.1158-1166, Mar. 2012.

[13] Avik Ray, Kanad Basu , Samir Biswas and M.K.Naskar, “ A Novel Distributed Algorithm for Topology Management in Mobile Ad-hoc Networks", International Conference on Computers and Devices for Communication (CODEC-06) Institute of Radio Physics and Electronics, University of Calcutta, 2006, pp.189192.

[14] Kiran K. Somasundaram, Kaustubh Jain, Vahid Tabatabaee and John S. Baras, " Distributed Topology Control for Stable Path Routing in Mobile Ad Hoc Networks", ISR Technical Report, 2010, pp.1-12.

[15] Liang Zhao, Errol L. Lloyd and S. S. Ravi, "Topology Control in Constant Rate Mobile Ad Hoc Networks", Wireless Networks, Springer, 2010, pp.1-15.

\section{AUTHORS PROFILE}

Mrs. ASHA T.S is presently working as the Associate professor in N S S College of Engineering, Palakkad. She has 20 years of teaching experiences and published one international journal and five national papers. She had taken her B.E Degree from The Indian Engineering College, Vadakkankulam in 1990. She has her M Tech degree in Opto Electronics and Laser technology from Cochin university of Science and Technology. Her research area include Wireless mobile communication and Optical communication.

Dr. N.J.R.Muniraj is presently working as a Principal of Tejaa Shakthi institute of Technology, Coimbatore. He has more than 23 years of teaching and five years of industrial experience. He has presented more than 40 National and International papers and published fifteen international journal papers. His research area includes VLSI Signal Processing, Neural Networks, Image Processing and MEMS. He is also heading the Tejaa Shakthi Innovation centre. 